\title{
Cyclooxygenase-1-derived PGE 2 promotes cell motility via the G-protein-coupled EP4 receptor during vertebrate gastrulation
}

\author{
Yong I. Cha, ${ }^{1}$ Seok-Hyung Kim, ${ }^{2}$ Diane Sepich,${ }^{2}$ F. Gregory Buchanan, ${ }^{1}$ Lilianna Solnica-Krezel, ${ }^{2,3}$ \\ and Raymond N. DuBois ${ }^{1,3,4}$ \\ ${ }^{1}$ Department of Medicine and Cancer Biology, Cell and Developmental Biology, Vanderbilt University Medical Center and \\ Vanderbilt-Ingram Cancer Center, Nashville, Tennessee, 37232-2279, USA; ${ }^{2}$ Department of Biological Sciences, Vanderbilt \\ University, Nashville, Tennessee 37235, USA
}

\begin{abstract}
Gastrulation is a fundamental process during embryogenesis that shapes proper body architecture and establishes three germ layers through coordinated cellular actions of proliferation, fate specification, and movement. Although many molecular pathways involved in the specification of cell fate and polarity during vertebrate gastrulation have been identified, little is known of the signaling that imparts cell motility. Here we show that prostaglandin $\mathrm{E}_{2}\left(\mathrm{PGE}_{2}\right)$ production by microsomal $\mathrm{PGE}_{2}$ synthase (Ptges) is essential for gastrulation movements in zebrafish. Furthermore, $\mathrm{PGE}_{2}$ signaling regulates morphogenetic movements of convergence and extension as well as epiboly through the G-protein-coupled PGE $_{2}$ receptor (EP4) via phosphatidylinositol 3-kinase (PI3K)/Akt. EP4 signaling is not required for proper cell shape or persistence of migration, but rather it promotes optimal cell migration speed during gastrulation. This work demonstrates a critical requirement of $\mathrm{PGE}_{2}$ signaling in promoting cell motility through the COX-1-Ptges-EP4 pathway, a previously unrecognized role for this biologically active lipid in early animal development.
\end{abstract}

[Keywords: Cancer; cell motility; cyclooxygenase; development; prostaglandin; zebrafish]

Supplemental material is available at http://www.genesdev.org.

Received September 13, 2005; revised version accepted November 14, 2005.

In mammals, prostaglandins (PG) have been implicated in a myriad of physiologic and pathologic processes, ranging from fundamental body functions such as reproduction to disease progression of inflammatory-mediated diseases like rheumatoid arthritis and cancer (Langenbach et al. 1999; Narumiya et al. 1999; Gupta and DuBois 2001). Prostaglandins are oxygenated metabolites of the 20-carbon polyunsaturated fatty acid molecule arachidonic acid, which is released from membrane phospholipids by the action of phospholipases. Cyclooxygenases (prostaglandin endoperoxide synthases, COX-1 and COX-2) catalyze the initial step in the prostaglandin biosynthesis, the conversion of arachidonic acid into $\mathrm{PGH}_{2}$ (Smith et al. 1996). Although COX-1 and COX-2 share similar structural and biochemical properties, they are encoded on separate chromosomes and exhibit distinct physiologic functions. Traditionally,

\footnotetext{
${ }^{3}$ These authors contributed equally to this work.

${ }^{4}$ Corresponding author.

E-MAIL raymond.dubois@vanderbilt.edu; FAX (615) 936-2697.

Article and publication are at http://www.genesdev.org/cgi/doi/10.1101/ gad.1374506.
}

COX-1 was thought to serve a homeostatic function, required for tissues to maintain a basal level of prostaglandins, and COX-2, the "inducible" isoform, is up-regulated by diverse stimuli including cytokines, mitogens, growth factors, and tumor promoters (Smith et al. 2000).

$\mathrm{PGH}_{2}$ is subsequently converted to one of several structurally related prostaglandins, $\mathrm{PGE}_{2}$ (prostaglandin $\mathrm{E}_{2}$ ), $\mathrm{PGD}_{2 \alpha}, \mathrm{PGF}_{2}, \mathrm{PGI}_{2}$ (prostaglandin $\mathrm{I}_{2}$ ), and thromboxane $\mathrm{A}_{2}\left(\mathrm{TxA}_{2}\right)$, by the activity of specific PG synthases (Negishi et al. 1995). In one such reaction, $\mathrm{PGH}_{2}$ is converted to $\mathrm{PGE}_{2}$ by microsomal prostaglandin $\mathrm{E}_{2}$ synthase (Ptges), a member of the MAPEG (membraneassociated proteins in eicosanoid and glutathione metabolism) family. $\mathrm{PGE}_{2}$ is then released from the cell and signals via binding to G-protein-coupled prostaglandin $\mathrm{E}_{2}$ receptors (EP1-4) embedded in the plasma membrane (Negishi et al. 1995). A large body of evidence implicates $\mathrm{PGE}_{2}$ as a lipid messenger that regulates a wide variety of important body functions in mammals, including pain and fever, maintenance of vascular tone, and cancer progression (Kobayashi and Narumiya 2002). However, deciphering the role of $\mathrm{PGE}_{2}$ in early mammalian development has been limited by the maternal contribution of 
prostaglandins in utero (Reese et al. 2000, 2001). As a result, the functional role for prostaglandins in early development remains virtually unknown.

Given these limitations in mammals, zebrafish is an emerging model for understanding the functional roles for prostaglandin signaling due to their extraembryonic development (Cha et al. 2005b). Previous studies in zebrafish demonstrated that the injection of antisense morpholino (MO) oligonucleotides designed to block the translation of cox1 transcripts leads to early gastrulation arrest, which is suppressed by coinjection of synthetic cox1 RNA (Grosser et al. 2002; Cha et al. 2005a). The gastrulation arrest phenotype was also recapitulated following treatment with pharmacologic inhibitors of cyclooxygenase, such as indomethacin or sulindac sulfide (Cha et al. 2005a). These observations prompted us to explore the specific prostaglandin signaling downstream of COX-1 during the early developmental process of gastrulation (Stern 2004).

\section{Results}

To understand the downstream effectors of COX-1 signaling during gastrulation, we initially analyzed the highly conserved zebrafish ortholog of $\mathrm{PGE}_{2}$ synthase, Ptges (Pini et al. 2005). The analysis of spatiotemporal expression revealed that ptges transcripts are maternally deposited and ubiquitously distributed during gastrulation, revealing that ptges and cox 1 transcripts colocalize during gastrulation (Fig. 1A,C). On the other hand, cox2 transcripts are not detected until after the end of gastrulation specifically in the anterior neuroectoderm (Fig. 1B). Analogous to other systems, these findings sug-
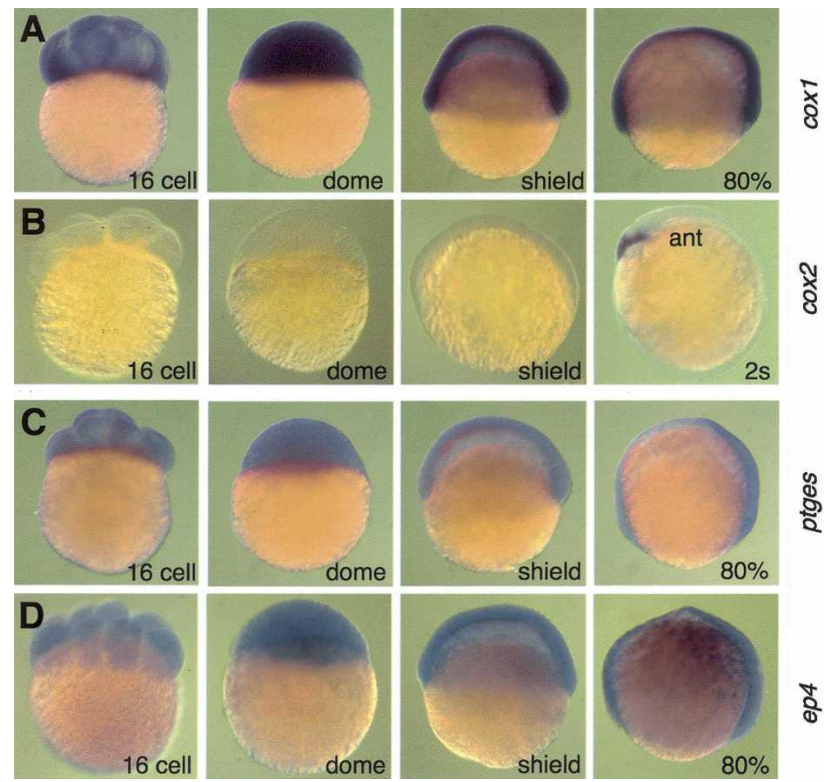

Figure 1. cox1, ptges, and ep4 transcripts are expressed ubiquitously during gastrulation. $(A-D)$ Lateral view, dorsal to the right. Expression of cox1 $(A), \operatorname{cox} 2(B)$, ptges $(C)$, and ep4 $(D)$ transcripts visualized by whole-mount in situ hybridization. (ant) Anterior. gested that the zebrafish Ptges functions downstream of COX-1 during gastrulation to generate $\mathrm{PGE}_{2}$. Accordingly measurements of prostaglandin levels using mass spectrometry revealed the production of $\mathrm{PGE}_{2}$ during gastrulation, along with $\mathrm{PGF}_{2 \alpha}$ (prostaglandin $\mathrm{F}_{2 \alpha}$ ) and $\mathrm{PGI}_{2}$ (Cha et al. 2005a), which parallels studies demonstrating the presence of $\mathrm{PGE}_{2}$ and $\mathrm{PGF}_{2 \alpha}$ in developing mammalian blastocysts (Davis et al. 1983).

To address the function of $\mathrm{PGE}_{2}$ signaling during zebrafish gastrulation, we designed two MOs to target ptges transcripts: One, ptges-MO1, is complementary to the $5^{\prime}$-untranslated region (UTR) and is expected to block translation, and the second, ptges-MO2, is complementary to the first intron-exon boundary and is expected to cause a splicing defect. Following ptges-MO1 or MO2 injections, we observed severe morphogenetic defects during gastrulation at a dose of 2 ng. Ptges-deficient embryos displayed a mispositioned head and shortened anteroposterior (AP) axis with delayed epiboly that completed only at tailbud or segmentation stage (Fig. 2B). After injection of a 4-ng dose of ptges-MO2, the embryos underwent complete gastrulation arrest similar to what we observed in COX-1-deficient embryos (Fig. 2C,E). To further characterize the morphological abnormalities associated with the loss of Ptges function, we analyzed the expression of several cell-type-specific markers at late gastrulation, anterior neuroectoderm (six3b) (Seo et al. 1999), mid-hindbrain boundary (pax2.1) (Krauss et al. 1991a), and axial and prospective posterior mesoderm (no tail/brachyury) (Schulte-Merker et al. 1994). While the transcripts of these markers were detected in Ptges-deficient embryos, their expression domains were shortened anteroposteriorly and widened mediolaterally (Fig. 2J,M). In addition, no tail expression in the nascent mesoderm revealed an enlarged blastopore. Marker analyses further suggested that Ptges-deficient embryos have defects in several gastrulation movements, including convergence, extension, and epiboly. Therefore, we hypothesized that Ptges-derived $\mathrm{PGE}_{2}$ plays an essential role in regulating cell movement during gastrulation.

To address whether lack of $\mathrm{PGE}_{2}$ production is solely responsible for these gastrulation defects, we tested whether the addition of exogenous $\mathrm{PGE}_{2}$, a downstream prostaglandin product of $\mathrm{PGE}_{2}$ synthase, could rescue the gastrulation phenotype in Ptges-deficient embryos. Ptges morphants were incubated in control media or media supplemented with $\mathrm{PGE}_{2}, \mathrm{PGF}_{2 \alpha}$, or $\mathrm{PGI}_{2}$, the predominant prostaglandins during zebrafish gastrulation. All morphants treated with $\mathrm{PGI}_{2}(n=55)$ (Fig. 2F) or $\mathrm{PGF}_{2 \alpha}(n=60)$ (Fig. 2G) exhibited gastrulation defects similar to untreated Ptges-deficient embryos. In contrast, $92 \%$ of morphants $(n=84)$ incubated in $\mathrm{PGE}_{2}$ (Fig. $2 \mathrm{H})$ completed epiboly and survived past tailbud stage (Supplementary Fig. 1), suggesting that Ptges regulates cell movement through secreted $\mathrm{PGE}_{2}$. Furthermore, we observed that the shapes of expression domains of pax2.1, six3b, and no tail were partially restored in Ptges morphants treated with $\mathrm{PGE}_{2}$ to those of uninjected control embryos (Fig. 2K,N,T,U). We also found that coin- 


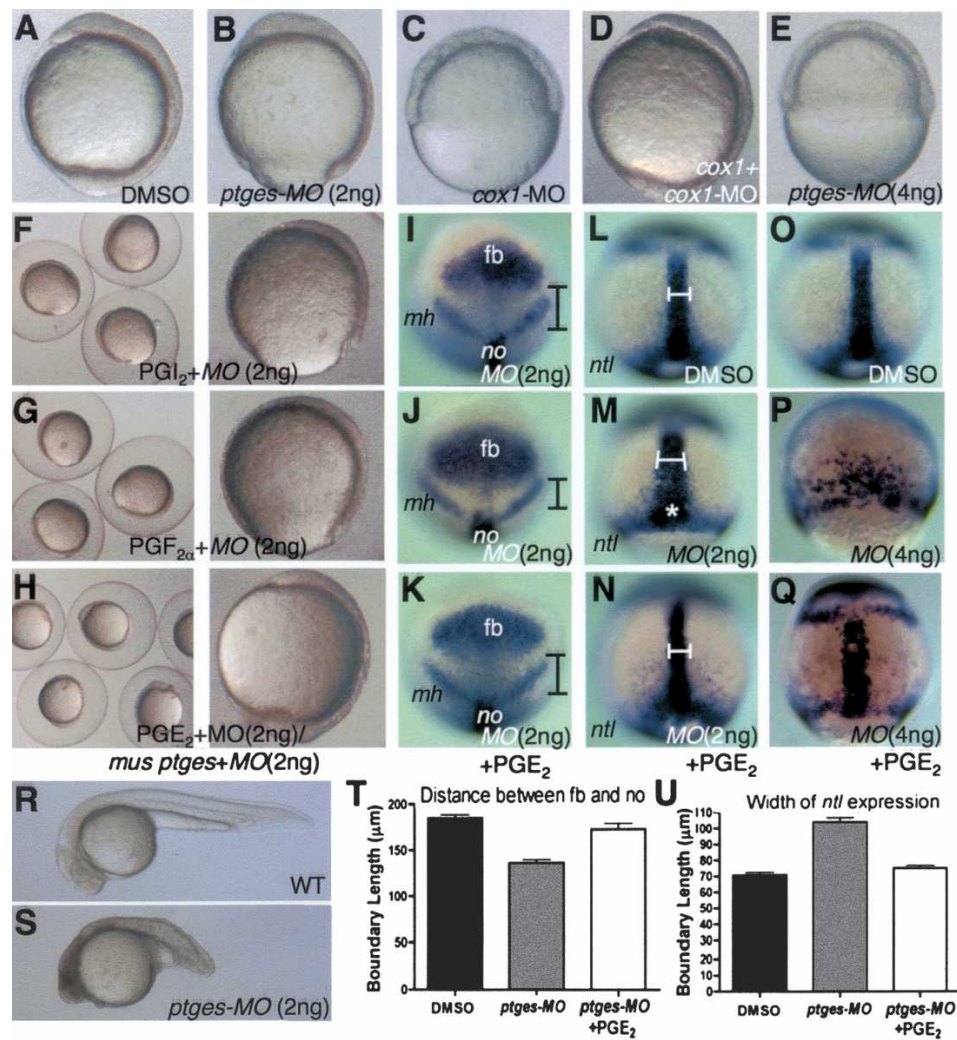

Figure 2. Ptges-deficient embryos exhibit gastrulation defects that are suppressed by coincubation with $\mathrm{PGE}_{2}$. $(A-H)$ Lateral view, dorsal to the right. $(I-K)$ Animal view, dorsal to the bottom. $(L-Q)$ Dorsal view, anterior to the top. $(R, S)$ Lateral view, anterior to the left. $(I-K)$ Boundaries indicate the distance from the posterior end of the forebrain to the anterior end of the notochord. $(L-Q)$ Boundaries indicate the width of the notochord. $(A, I, L, O)$ Control embryos treated with DMSO. $(B, F-$ $H, J, K, M, N, S)$ Embryos injected with 2 ng of ptges-MO2. $(E, P, Q)$ Embryos injected with 4 ng of ptges-MO2. $(C, D)$ Embryos injected with $10 \mathrm{ng}$ of cox1-MO. (D) Embryos coinjected with cox1-MO (10 ng) and cox1 RNA (50 pg). $(F)$ Embryos injected with $2 \mathrm{ng}$ of ptges-MO2 $+\mathrm{PGI}_{2} \cdot(G)$ Embryos injected with $2 \mathrm{ng}$ of ptges-MO2 $+\mathrm{PGF}_{2 \alpha}$. $(H, K, N)$ Embryos injected with 2 ng of ptges$M O 2+\mathrm{PGE}_{2}$. (Q) Embryos injected with 4 ng of ptges$\mathrm{MO} 2+\mathrm{PGE}_{2}$. $(H)$ Embryos coinjected with ptges-MO2 $(2 \mathrm{ng})+$ mouse ptges RNA (100 pg). (I-K) Expression patterns of six3b, pax2.1. (L-Q) Expression pattern of no tail. (T) Quantitative analyses of the distance between the forebrain $(\mathrm{fb})$ and notochord (no) in DMSO, ptges$M O 2$, and ptges-MO2 $+\mathrm{PGE}_{2}(n=10$ per each group). $(U)$ Quantitative analyses of width of $n t l$ expression in DMSO, ptges-MO2, and ptges-MO2 + $\mathrm{PGE}_{2}(n=10$ per each group). (fb) Forebrain; (mh) mid-hindbrain; (no) notochord. The white star marks the blastopore to illustrate delayed epiboly. For details see text. jecting mouse Ptges RNA (50 pg) suppressed the gastrulation defects in Ptges morphants in $75 \%$ of embryos $(n=120)$ (Fig. 2H; Supplementary Fig. 1), further demonstrating the specificity of the ptges-MOs. In addition, the gastrulation arrest in embryos injected with a 4-ng dose of ptges-MO2 was also partially suppressed by $\mathrm{PGE}_{2}$ treatment (Fig. 2P,Q). We also tested the effectiveness of the ptges-MO. We observed that injection of 2 or $4 \mathrm{ng}$ of ptges-MO2, targeting the first intron-exon boundary, results in truncation of the transcript size by $\sim 82$ base pairs (bp) due to deletion of exon 2 (Supplementary Fig. 2A). Since this splicing MO targets the beginning of exon 2, the splicing machinery targets the splicing donor site of intron 1 and the acceptor site of exon 3 to cause deletion of exon 2. In addition, some wild-type transcripts remain in embryos injected with $2 \mathrm{ng}$, but not in embryos injected with $4 \mathrm{ng}$ of ptges-MO2, further demonstrating that injection of $2 \mathrm{ng}$ of ptges-MO2 represents a hypomorphic situation (Supplementary Fig. 2A). Interestingly, overexpression of ptges RNA did not lead to any observable gastrulation abnormalities. This is also consistent with our previous finding that incubation of wildtype embryos with high doses of $\mathrm{PGE}_{2}$ or overexpression of COX-1 didn't cause any noticeable defects during development (Cha et al. 2005). The remarkable ability of $\mathrm{PGE}_{2}$ to overcome the gastrulation defect in Ptges morphants and normal gastrulation in embryos with excess $\mathrm{PGE}_{2}$ indicate that this lipid messenger plays an essential but permissive role in gastrulation movements of convergence and extension as well as epiboly.

Next we sought to identify downstream effectors of
$\mathrm{PGE}_{2}$ during gastrulation. In mammals, EP receptors (EP1-4), members of the G-protein-coupled receptor (GPCR) family, bind to $\mathrm{PGE}_{2}$ with high affinity to activate downstream signals (Breyer et al. 2001). We searched the NCBI database and identified zebrafish ESTs with the highest sequence similarities to mammalian EP receptors, designated as Ep2 and Ep4 (Supplementary Fig. 3). Spatiotemporal analysis of ep2 and ep4 expression demonstrated ubiquitous distribution of ep4 transcripts in early embryos before the onset of the zygotic transcription and also later throughout gastrulation, while ep2 RNA was not detected until the end of gastrulation. RT-PCR analysis confirmed the presence of ptges and ep4, but not ep2, transcripts during gastrulation (Fig. 1D; data not shown). To address the role of Ep2 and Ep4 receptors in early embryonic development, we designed two MOs targeting ep2 and ep4 receptor transcripts (MO1 targets 5'-UTR, while MO2 targets the start codon), to block their translation. Embryos injected with up to $15 \mathrm{ng}$ of ep2-MO1 or ep2-MO2 failed to demonstrate any effect on gastrulation, consistent with the lack of expression during early development. However, injection of $2 \mathrm{ng}$ of ep4-MO1 or ep4-MO2 produced embryos with vegetally shifted heads and shortened AP axes at the end of the gastrula period $195 \%$ of embryos displayed this phenotype, $n=130$ ) (Fig. 3D,E). The shortening of the AP axis was observed throughout the somitogenesis stages, and at $24 \mathrm{~h}$ post-fertilization (hpf), EP4deficient embryos exhibited a shortened AP axis, laterally elongated somites, and wavy notochord (Fig. $3 F)$. This phenotype resembles that of mutants in the 


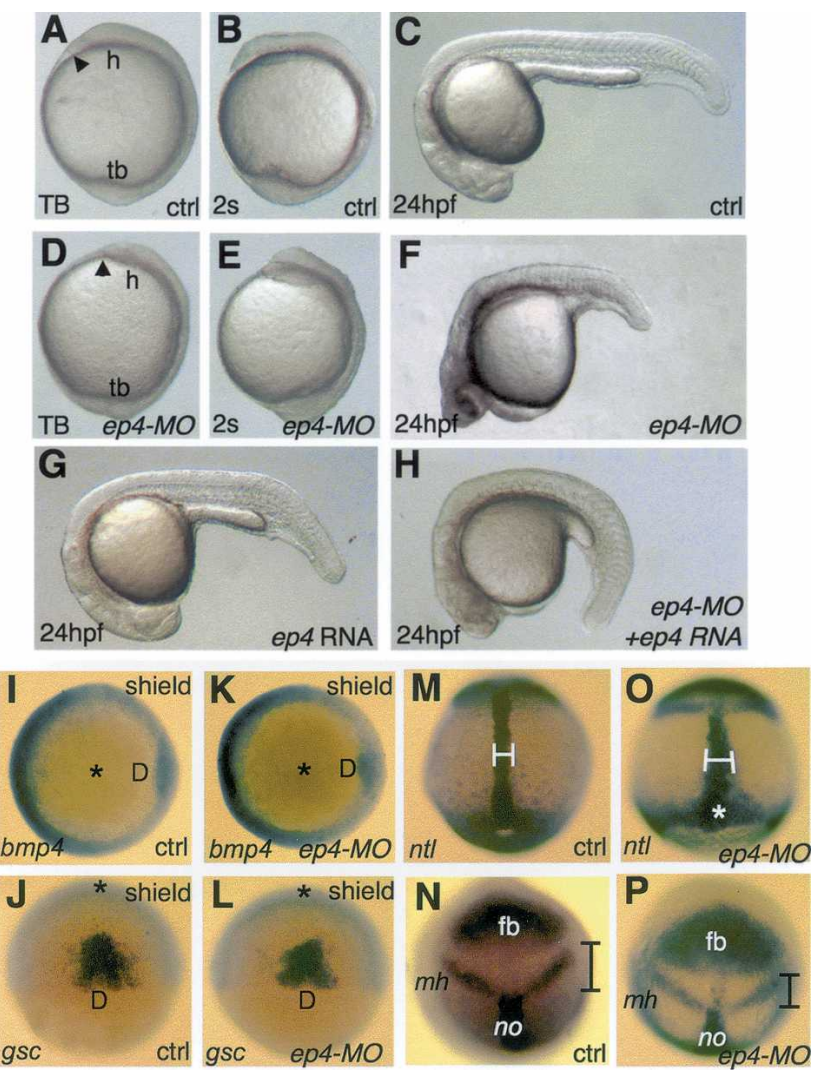

Figure 3. EP4-deficient embryos exhibit convergence and extension defect during gastrulation. $(A, B, D, E)$ Lateral view, dorsal to the right. $(C, F, G, H)$ Lateral view, anterior to the left. $(I, K)$ Animal view, dorsal to the right. $(J, L)$ Dorsal view, animal pole to the top. $(M, O)$ Dorsal view, anterior to the top. $(N, P)$ Anterior view, dorsal to the bottom. $(M, N)$ Boundaries indicate the width of the notochord. $(N, P)$ Boundaries indicate the distance from the posterior end of the forebrain to the anterior end of the notochord. $(A-C, I, J, M, N)$ Uninjected control. $(D-F, H, K, L, O, P)$ ep4-MO2 (2 ng) injected embryos. $(G, H)$ ep4 RNA (80 pg) injected embryos. $(H)$ Embryos injected with ep4-MO2 (2 ng) + ep4 RNA (80 pg). $(I, K)$ bmp4 expressions. $(J, L)$ gsc expressions. $(M, O)$ no tail (ntl) expressions. $(N, P)$ six3, pax2, and $n t l$ expressions. (h) Head; (tb) tailbud; (D) dorsal; (fb) forebrain; (mh) mid-hindbrain; (no) notochord. The arrowhead points to the location of the prechordal plate at the tailbud stage. The white star highlights the blastopore to illustrate delayed epiboly. The black star indicates the animal pole.

noncanonical Wnt/planar cell polarity (PCP) pathway defective in convergence and extension gastrulation movements, such as knypek (Topczewski et al. 2001), trilobite (Jessen et al. 2002), and pipetail (wnt5) (Rauch et al. 1997). Next, we determined whether ptges-MO and ep4$M O$ demonstrate synergism. Coinjection of suboptimal dose of ep4-MO2 (1 ng) with ptges-MO2 (1 ng) produced embryos with a more severely shortened body axis at 24 hpf as compared with those embryos injected with a 1-ng dose of either ep4-MO or ptges-MO2 (Supplementary Fig. 2C). This result is consistent with Ptges and Ep4 functioning in the same genetic pathway.
To understand better the morphological differences in EP4-deficient embryos, we examined the expression of several cell-type-specific genes. The expression of goosecoid (gsc), a presumptive prechordal mesoendoderm marker, and bmp4, a marker of ventroposterior tissues, was similar to that in uninjected sibling embryos at the shield stage, indicating normal patterning during early gastrulation (Fig. 3K,L). Next we found that dorsal midline expression of no tail was widened and its blastopore expression domain animally shifted (Fig. 3O), while the expression domains of neural markers were shortened in the AP axis (Fig. 3P), reminiscent of the convergence, extension, and epiboly defects in Ptges-deficient embryos. However, in contrast to Ptges- and COX-1-deficient embryos, and consistent with the function of EP4 downstream of $\mathrm{PGE}_{2}$, gastrulation defects of EP4 morphants were not suppressed by exogenously supplied $\mathrm{PGE}_{2}(0 \%, n=55)$. To test the efficiency by which ep4$M O$ inhibit Ep4 function, we designed an Ep4-GFP construct. We observed that coinjection of synthetic ep4GFP RNA with ep4-MO2 resulted in a loss of GFP expression compared with embryos injected with ep4-GFP RNA alone (Supplementary Fig. 2B). These experiments support the argument that the ep4-MO leads to a strong Ep4 loss of function, consistent with the inability of $\mathrm{PGE}_{2}$ to suppress gastrulation defects. Injection of synthetic ep4 RNA at 80 pg led to a mild epiboly and convergence and extension defects and ultimately resulted in a shortened tail at $24 \mathrm{hpf}$ (Fig. 3G). In addition, coinjection of this ep4 RNA with ep4-MO2 suppressed the strong convergence and extension phenotype of EP4-deficient embryos $152 \%$ of embryos had partially restored wild-type phenotype at $24 \mathrm{hpf} ; n=72$ ), demonstrating the specificity of the ep4-MO2. However, injection of ep4 RNA at higher levels led to a dose-dependent defect in convergence and extension (data not shown) and did not suppress the morphant phenotype in rescue experiments. Previously it has been shown that genes that regulate cell movement often have similar phenotypes in loss-of-function (LOF) or gain-of-function (GOF) experiments (Jessen et al. 2002; Carreira-Barbosa et al. 2003; Lin et al. 2005), suggesting that the levels of these gene products must be tightly regulated. These results show that EP4 receptors, similar to Ptges, regulate cell movements without altering patterning and fate specification. In addition, EP4 specifically acts as a receptor for the Ptges-derived $\mathrm{PGE}_{2}$ to regulate cell movement during gastrulation.

The morphological abnormalities observed in Ptgesand EP4-deficient embryos are consistent with convergence, extension, and epiboly defects. To identify cell behaviors that depend on EP4 receptor function, we performed Nomarski time-lapse analyses of lateral mesoderm cells in wild-type (two embryos, 84 cells), control ep4-MO-injected (EP4-MOc) (two embryos, 80 cells), and ep4-MO2-injected (six embryos, 224 cells) embryos at mid-gastrulation. These moderately elongated cells undergo directed migration toward the dorsal midline along complex trajectories (Jessen et al. 2002). Analysis of total movement speed, accounting for the cell movement in 
all directions, showed that cells in EP4-deficient embryos had reduced total speed (75\% of the control level). In addition, the net dorsal speed was also attenuated to a similar degree, which was $67 \%$ of the control level (Fig. 4D; Supplementary Movies 1-3). By comparing the meandering index, a ratio of total to the net path, which measures the tendency of cells to deviate from their directional path, we found that the lateral mesodermal cells in EP4-deficient embryos largely migrate toward the dorsal midline along trajectories similar to the control cells (wild type $=1.29, n=84$; EP4-deficient embryos $=1.19, n=224 ; p<0.57$ ) (Figs. 4E, 5), indicating that the directional cell movement in EP4-deficient embryos is not significantly compromised. In addition, we found that the shape of cells in this population, as determined by the length-to-width ratio (LWR), remains relatively unchanged in EP4 morphants, as compared with ep4-MOc-injected or uninjected wild type (Fig. 4F) at mid-gastrulation. The normal cell shape and meandering index associated with defective convergence movements of lateral mesodermal cells in EP4-deficient embryos contrast cellular defects reported for embryos with de-

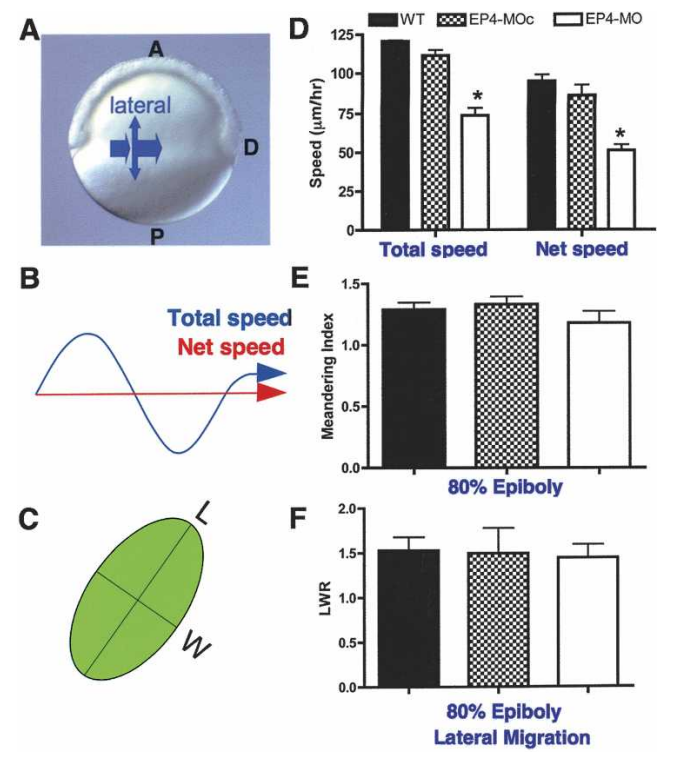

Figure 4. EP4-deficient embryos exhibit decreased cell motility in dorsal migration, while maintaining normal cell shape. (A) Domains of convergence and extension movements in zebrafish gastrulae (Myers et al. 2002b). Orange arrows indicate domains of slow convergence and extension. $(B)$ A diagram to demonstrate the difference between total versus net speed. $(C) \mathrm{A}$ schematic representation of the method used to measure cell shape. (LWR) Length-to-width ratio. $(D-F)$ Cell behavior of mesoderm cells during slow convergence toward the dorsal midline. (D) Total and net dorsal speed of lateral mesodermal cells at $80 \%$ epiboly. (E) Meandering index of lateral mesodermal cells. The meandering index was determined by dividing the total speed by the net speed. $(F)$ LWR of lateral mesodermal cells. Lateral mesoderm measurements were made in wild-type (two embryos, 84 cells), control EP4-MO-injected (EP4-MOc; two embryos, 80 cells), and EP4-deficient (six embryos, 224 cells) embryos.

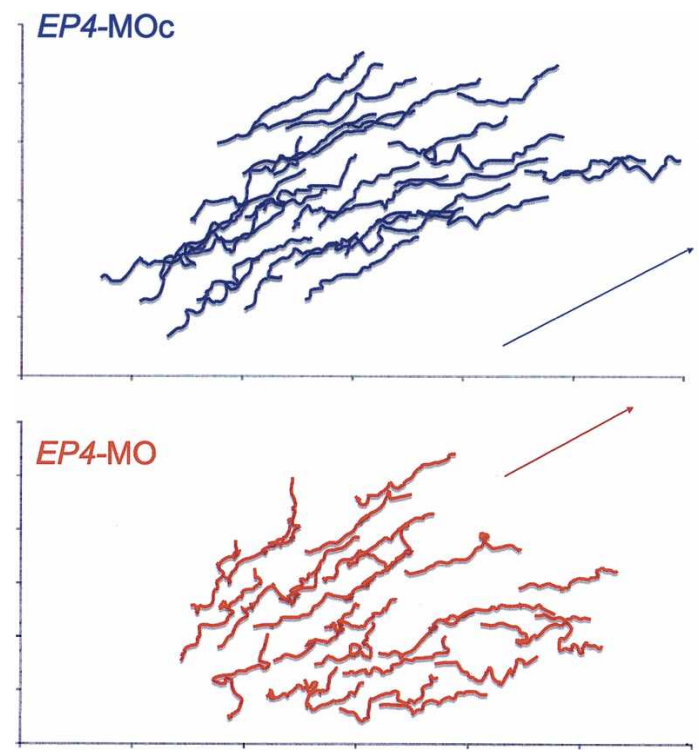

Figure 5. EP4-deficient embryos exhibit reduced net path, but not persistence of directional movement, during dorsal migration. Representation of the paths traveled by lateral mesodermal cells at $80 \%$ epiboly in embryos injected with control EP4-MO (EP4-MOc) and EP4-MO. The path of each individual cell movement from the lateral mesoderm region was plotted over 40 min, starting at $80 \%$ epiboly. Colored arrows demonstrate the vector representation of the average direction and net length of cells from EP4-MOc (blue) and EP4-deficient (red) embryos.

fective G $\alpha 12 / 13$ signaling (Lin et al. 2005). In this situation, impaired convergence of lateral mesodermal cells has been linked to rounder cell shapes and less persistent dorsal movement. Therefore, given the above observations and impaired epiboly in COX-1-, Ptges-, and EP4deficient embryos, these studies provide evidence that $\mathrm{PGE}_{2}$ signaling primarily regulates motility of mesodermal cells during gastrulation, without significantly altering cell shape or persistence of migration.

We have previously shown that $\mathrm{PGE}_{2}$ can affect cancer metastasis by increasing proliferation, migration, and invasiveness of cancer cells and the stimulatory effects of $\mathrm{PGE}_{2}$ are dependent on the activation of the phosphatidylinositol 3-kinase (PI3K)/Akt pathway (Sheng et al. 2001; Buchanan et al. 2003). The serine/threonine kinase Akt, or protein kinase $\mathrm{B}(\mathrm{Akt} / \mathrm{PKB})$ is a direct downstream effector of PI3K. Akt/PKB lies at the crossroad of multiple cellular signaling pathways and acts as a transducer of inputs initiated by receptor signaling cascades that activate PI3K (Katso et al. 2001). Embryos treated with the PI3K inhibitor LY294002 exhibited abnormal morphology consistent with defects in convergence and extension, as well as epiboly during gastrulation (Fig. 6B), mimicking phenotypes of Ptges- and EP4-deficient embryos. To test whether PI3K/Akt activation is mediated by the EP4 receptor, we determined the phosphorylation status of Akt in embryos injected with MOs against ep2, ep4, and ptges transcripts. The zebrafish homolog of Akt has been cloned previously, and was shown to exhibit the phosphorylation sites identified in human 
Figure 6. PI3K/Akt is activated downstream of EP4 signaling. $(A-C)$ Lateral view, dorsal to the right. (B) Ten micromolar PI3K/Akt inhibitor, LY294002. (C) Thirty micromolar PI3K/Akt inhibitor, LY294002. (D) Uninjected, ep4-MO2 (2 $\mathrm{ng})$, or ptges-MO2 (2 ng) injected. (E) Uninjected, ep4-MO2 (2 ng), or ep2-MO2 (2 ng) injected. (F) Uninjected or ep4-MO2-injected (2 $\mathrm{ng}$ ) embryos treated with or without exogenous $\mathrm{PGE}_{2}(5 \mu \mathrm{M})$. $(G)$ Uninjected, ep4-MO2 (2 ng), or ep2-MO (2 ng) injected embryos. (H) Molecular mechanisms for $\mathrm{PGE}_{2}$ signaling in vertebrate gastrulation. Lysates from 2.5 embryos were loaded into each well for Western blot analyses. (pAKT) Phosphorylated AKT; (AKT) total AKT; (pERK) phosphorylated ERK; (ERK) total ERK. Please see text and Materials and Methods for details.
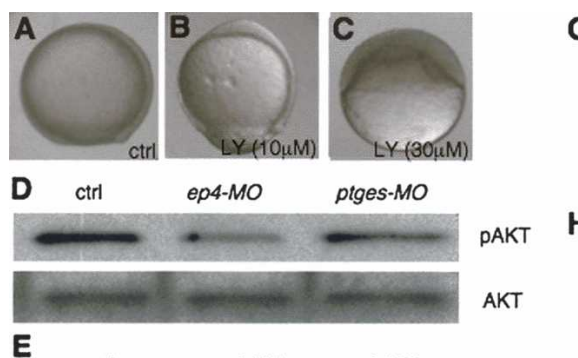

E cirl

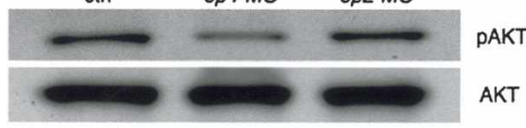

F

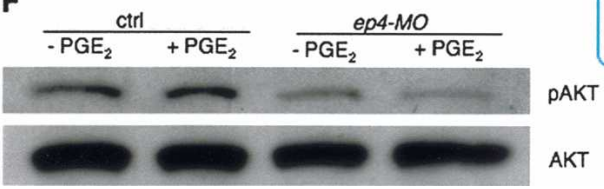

G

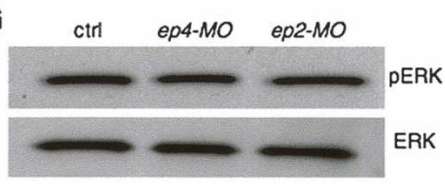

H

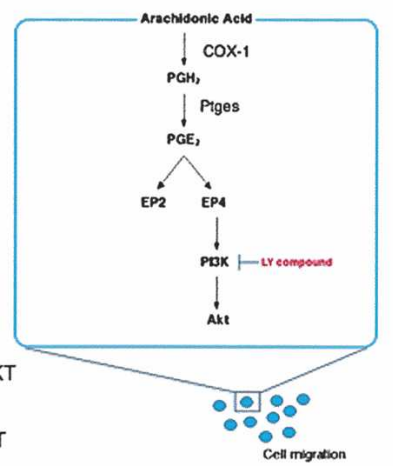

Akt, Thr308, and Ser473 (Chan et al. 2002). In both EP4and Ptges-deficient embryos, we observed a significant decrease in levels of the phosphorylated form of Akt (Fig. $6 \mathrm{D}, \mathrm{E})$, while the total Akt levels were constant. Consistent with a permissive role of $\mathrm{PGE}_{2}$, the embryos with high levels of $\mathrm{PGE}_{2}$ did not result in increased phosphorylation of Akt (Fig. 6F). We also tested the possibility that the ERK pathway, as shown previously (Fujino et al. 2003), can mediate the EP4 signaling and found that ERK signaling is not affected in EP4-deficient embryos (Fig. 6G), showing the restriction of EP4 signaling to the PI3K/Akt pathway. Taken together, our data suggest that the EP4 receptor acts upstream of the PI3K/Akt pathway to regulate cell movement during gastrulation (Fig. 6H).

\section{Discussion}

Previous studies in mice have not revealed much about the role for prostaglandins in early development. In heterozygous matings, Cox-1-null mice were born in the expected ratios and lived a normal life span (Langenbach et al. 1995). This finding was quite surprising given the putative "housekeeping" function of the COX-1 enzyme. Although Cox-2-null mice demonstrated numerous developmental defects (Dinchuk et al. 1995), their embryogenesis proceeded normally. Given the vital role of prostaglandins in normal physiologic settings, it has been hypothesized that the maternal contribution of prostaglandins in the placenta allows the prostaglandindeficient embryos to develop normally (Reese et al. 2000, 2001). Consistent with this notion, mammalian blastocysts produce high levels of $\mathrm{PGE}_{2}$ and $\mathrm{PGF}_{2 \alpha}$ (Dey et al. 1980; Davis et al. 1983), and murine blastocysts cultured outside of the uterus do not survive in the presence of COX inhibitors (H. Wang and S.K. Dey, pers. comm.). Therefore, the functional role of prostaglandins and the signaling cascades they regulate in early development have been virtually unknown.

By using the externally developing zebrafish embryos as a model system, we circumvented the maternal interference, allowing us to study the functional role for endogenous prostaglandins. Our data conclusively demonstrate the conservation of the $\mathrm{PGE}_{2}$ signaling pathway in vertebrates. Previous studies in zebrafish demonstrated that COX-1-derived prostaglandins may be important for early development (Grosser et al. 2002; Cha et al. 2005). Here we show that prostaglandins during gastrulation are produced mainly by COX-1, since COX-2 expression does not take place until after the end of gastrulation. Furthermore, we demonstrate that expression of Ptges colocalizes with that of COX-1 and the COX-1-Ptges pathway governs the production of $\mathrm{PGE}_{2}$ during gastrulation. Previous work showed that COX-1 is coupled to cytosolic $\mathrm{PGE}_{2}$ synthase (cPGES), while COX-2 is colocalized with microsomal $\mathrm{PGE}_{2}$ synthase (Ptges) (Takeda et al. 2004) in gastrointestinal hamartomas. In zebrafish, Ptges colocalizes with COX-1 during gastrulation, but is coexpressed with both COX isoforms during somitogenesis in the posterior intermediate mesoderm and anterior neuroectoderm (Y.I. Cha, L. Solnica-Krezel, and R.N. DuBois, unpubl.), suggesting that Ptges may be the predominant source of $\mathrm{PGE}_{2}$ during embryogenesis. Consistently, when we injected antisense morpholino oligonucleotides targeting cytosolic $\mathrm{PGE}_{2}$ synthase in zebrafish, we didn't observe any phenotypic changes during gastrulation or thereafter (data not shown). This finding is similar to the situation reported in mice, where inactivation of mouse Ptges reduces inflammatory responses to carrageenan, similar to the effects of NSAIDs, while the cPGES-derived $\mathrm{PGE}_{2}$ is relatively unimportant (Trebino et al. 2003), suggesting that Ptges may also be the dominant source of $\mathrm{PGE}_{2}$ during inflammation. Taken together, our data suggest that the COX-1-Ptges pathway may be solely responsible for providing basal levels of $\mathrm{PGE}_{2}$ during early development.

During vertebrate gastrulation, changes in the levels of proteins that regulate cell movement can impair gastrulation in either LOF or GOF experiments (Jessen et al. 
2002; Carreira-Barbosa et al. 2003; Lin et al. 2005). Similarly, LOF or GOF experiments with EP4 receptor both result in gastrulation defects, while overexpression of ptges or treatment with $\mathrm{PGE}_{2}$ does not produce a noticeable change in gastrulation phenotype. In addition, the phosphorylation status of Akt in wild-type embryos treated with $\mathrm{PGE}_{2}$ remains unaltered (Fig. 6F). These data suggest that $\mathrm{PGE}_{2}$ plays an essential, but permissive, role during vertebrate gastrulation. It remains to be determined whether the excess EP4 receptor impairs gastrulation by increased normal signaling or functions by an antimorphic or neomorphic activity.

One of the more interesting questions that arises from this study is why does the ptges-MO cause an even stronger phenotype than the one observed in EP4 morphants? From cell culture experiments, we know that $\mathrm{PGE}_{2}$ can exert effects that are not totally dependent on the EP4 receptor. For example, we have previously reported that $\mathrm{PGE}_{2}$ can indirectly transactivate the peroxisome proliferator-activated receptor $\delta$ (PPAR $\delta$ ) receptor in colorectal adenoma growth in APC ${ }^{\text {min }}$ mice (Wang et al. 2004). Therefore, we hypothesize that during zebrafish gastrulation, loss of $\mathrm{PGE}_{2}$ can lead to a stronger phenotype than that exhibited by EP4 morphants due to a disruption of processes independent of the EP4 pathway. In support of this, there are differences in Ptges and EP4 morphant phenotypes at 24 hpf (Figs. 2S, 3F). However, our data convincingly demonstrate that the effects of $\mathrm{PGE}_{2}$ on cell movement are dependent specifically on the EP4 receptor.

Another intriguing concept that arises from this study is the conserved role of the EP4-PI3K/Akt pathway in regulation of cell movement. Previous work in zebrafish demonstrated that PI3K/Akt signaling is required downstream of PDGF for proper cell shape, process formation, and movement during anterior migration of prechordal mesodermal cells (Montero et al. 2003). However, our studies also implicate PI3K/Akt signaling in proper convergence, extension, and epiboly processes. We provide two lines of evidence that suggest PI3K/Akt lies downstream of $\mathrm{PGE}_{2}$ during gastrulation: First, we observed decreased activation of Akt in EP4-deficient embryos. Second, loss-of-function phenotypes in Ptges- and EP4deficient embryos share similar defects in convergence, extension, and epiboly to those of embryos treated with a PI3K inhibitor. With respect to gastrulation cell behaviors that depend on this pathway, our cell tracking data support the notion that EP4 receptor signaling uses PI3K/Akt to promote cell motility, rather than cell shape or persistence of migration. Consistently, zebrafish primordial germ cells also depend on PI3K signaling for cell motility during their migration (Dumstrei et al. 2004), while PI3K/Akt signaling has been implicated also in cell movement and directionality during neutrophil migration (Stephens et al. 2002; Wang et al. 2002). These observations suggest that PI3K/Akt is a key regulator of distinct properties of moving cells in various cellular contexts. Therefore, we cannot exclude the possibility that EP4 signaling may regulate other aspects of cell behavior during zebrafish gastrulation and development.
So, how does the EP4 receptor activate the PI3K/Akt pathway? As mentioned earlier, the EP4 receptor is a member of the GPCR superfamily. Previous studies in mammalian cell culture demonstrated that G $\beta \gamma$ dimers transmit signals from GPCR to a variety of intracellular effectors in distinct cellular contexts (Schwindinger and Robishaw 2001). One of the targets of G $\beta \gamma$ dimers involves direct activation of PI3K by binding of specific G $\beta \gamma$ dimers to both subunits of PI3K (Leopoldt et al. 1998). Whether G $\beta \gamma$ dimers can transduce EP4 signaling still remains to be seen both in mammalian settings as well as during zebrafish gastrulation. However, our perusal through the zebrafish genome revealed that the presence of multiple human G $\beta$ and $G \gamma$ homologs and their role during zebrafish gastrulation have not yet been elucidated.

Although $\mathrm{PGE}_{2}$ signaling has been shown to promote migration and invasive behaviors of several cancer cell lines in vitro (Sheng et al. 2001; Buchanan et al. 2003), the cell movement behaviors and molecular pathways regulated by $\mathrm{PGE}_{2}$ signaling in vivo are not known. Our studies provide the first evidence that $\mathrm{PGE}_{2}$ signaling regulates cell movement in vivo by promoting cell motility during gastrulation. Understanding the detailed mechanism of $\mathrm{PGE}_{2}$ signaling in zebrafish gastrulation may ultimately provide significant insights into how $\mathrm{PGE}_{2}$ regulates various processes that require cell movement, including metastatic spread of cancer.

\section{Materials and methods}

\section{Zebrafish maintenance, embryo generation, and staging}

$\mathrm{AB}^{\star}$ and $\mathrm{TL}$ wild-type zebrafish strains were maintained as described (Solnica-Krezel et al. 1996). Embryos were obtained from natural spawnings and staged according to morphology as described (Kimmel et al. 1995).

\section{Cloning of prostaglandin $E_{2}$ receptors and synthases}

An expressed sequence tag (EST) with sequence similarity to human EP2 and EP4 receptors were isolated by PCR (Advantage2; Clontech), and cloned into the pCR2.1 vector (Invitrogen) and used for antisense probe synthesis with SP6 RNA polymerase. For misexpression, the full-length cDNA was cloned into the pCS2+ vector and used for capped RNA synthesis with SP6 RNA polymerase after NotI linearization (mMESSAGE mMACHINE; Ambion). The NCBI accession numbers for EP2 and EP4 are DQ286580 and DQ202321, respectively. To obtain full-length prostaglandin $\mathrm{E}_{2}$ synthases, we designed primers against previously isolated sequences of ptges and cpges (Pini et al. 2005) and isolated them by PCR (Advantage2; Clontech). Full-length PCR products were cloned into the pCR2.1 vector (Invitrogen) and used for antisense probe synthesis with SP6 RNA polymerase.

\section{In situ hybridization}

Antisense probes for zebrafish $\operatorname{cox} 1$ and $\operatorname{cox} 2$ were synthesized as described (Grosser et al. 2002). Antisense RNA probes were synthesized from cDNAs encoding six3b (Kobayashi et al. 1998), nt1 (Schulte-Merker et al. 1992), and pax2.1 (Krauss et al. $1991 b \mid$. Whole-mount in situ hybridization was performed ac- 
cording to Marlow et al. (2002). Embryos were analyzed on a Zeiss Axioplot, and images were captured with the Nikon Coolpix 4500. Each in situ experiment was done at least twice, using $\sim 20$ embryos.

\section{MO design and MO/RNA injections}

MO design and MO/RNA injections were performed at the one-cell stage as described (Marlow et al. 2002). MOs were designed to the predicted start codon (MO1) and first exon-intron boundary (MO2) of ptges (underline indicates the predicted start codon): ptges-MO1 (5'-TCAGCAAAAAGTTACACTCT CTCAT-3') and ptges-MO2 (5'-GTTTTGTGCTCTTACCTCC TACAGC-3'). For EP receptors, we designed two MOs against the 5'-UTR (MO1) and the predicted start codon (MO2): ep2MO1 (5'-GATGTTGGCATGTTTGAGAGCATGC-3'), ep2-MO2 (5'-ACTGTCAATACAGGTCCCATTTTC-3'), ep4-MO1 (5'CG CGCTGGAGGTCTGGAGATCGCGC-3'), ep4-MO2 (5'-CACGGTGGGCTCCATGCTGCTGCTG- $\left.3^{\prime}\right)$, and ep4-MOc (5' -CAt GGTGGcgTgCATGCTaCTGCTG-3'; lowercase letters denote mutated sites). All MOs were obtained from Gene Tools, LLC. Zebrafish ep4 sense-capped RNA was synthesized using mMessage Machine (Ambion) after template linearization with enzyme. For phenotype rescue and phenocopy experiments, $80 \mathrm{pg}$ of ep4 RNA and 2 ng of ep4-MO2 per embryo were used.

\section{Prostaglandin rescue experiments}

For prostaglandin supplementation experiments, we used commercially available prostaglandins (Cayman), $\mathrm{PGE}_{2}, \mathrm{PGF}_{2 \alpha}$, and $\mathrm{PGI}_{2}$. We supplemented Ptges-deficient embryos with $5 \mu \mathrm{M}$ PGs in $1 \%$ DMSO egg media at the beginning of the epiboly process as previously described (Cha et al. 2005).

\section{Nomarski time-lapse analysis}

Images of lateral gastrula mesodermal cells at mid-gastrulation (80\% epiboly) were carried out as described previously (Myers et al. 2002a). Cell movement measurement data obtained in Object-Image (NIH image) were exported to Excel (Microsoft), where cell migration speed, path, direction, turning angle, and LWRs were determined. The movement direction of lateral mesodermal cells was determined at 1-min intervals.

\section{Inhibitor treatment}

To block PI3K and PDGF activity, we used LY294002 (Calbiochem), a specific inhibitor of PI3K activity (Vlahos et al. 1994). Embryos were usually treated from $30 \%$ epiboly to the YPC stage in 10 or $30 \mu \mathrm{M}$ LY294002 to monitor the phenotypes.

\section{Western blot analysis}

Embryos were injected with ptges-MO, ep2-MO2, or ep4-MO2, or treated with LY. Endogenous levels of Akt and ERK activation were measured. Embryos were injected with a 2-ng dose of ptges-MO, ep2-MO2, ep4-MO2 wild type at the one-cell stage, or treated with $\mathrm{PGE}_{2}$ starting at the $30 \%$ epiboly stage. Embryos were then collected between the $80 \%$ and $90 \%$ epiboly stage, dechorionated manually, and homogenized in $50 \mathrm{mM}$ HEPES (pH 7.5), $150 \mathrm{mM} \mathrm{NaCl}, 1 \%$ Triton X-100. Equal amounts of samples were separated by SDS-PAGE and transferred to polyvinylidene difluoride membranes. The membranes were blocked in TTBS (Tris-buffered saline with $0.1 \%$ Tween 20) containing either $5 \%$ dry milk or BSA. Primary antibody incubations were performed in TTBS with either $5 \%$ dry milk or
BSA overnight at $4^{\circ} \mathrm{C}$. After washing, the membranes were incubated with the appropriate secondary peroxidase-conjugated antibody for $1 \mathrm{~h}$ in TTBS with either $5 \%$ dry milk or BSA. Immunoreactive proteins were visualized using the enhanced chemiluminescence system from Amersham Biosciences. Antibodies to Akt, Erk, phospho-Akt, and phospho-Erk were obtained from Cell Signaling.

\section{Acknowledgments}

We thank the following individuals for their help with this work: S.K. Dey, Erez Raz, and Michael Backlund for critical reading of the manuscript. This work in the DuBois laboratory is supported in part from the United States Public Health Services Grants RO-DK-62112 and P0-CA-77839. R.N.D. is the B.F. Byrd Professor of Molecular Oncology and the recipient of an NIH MERIT award (R37-DK47297). The work in the L.S-.K. laboratory was funded by R01GM55101. This research has been supported in part by the Zebrafish Initiative funded by the Vanderbilt University Academic Venture Capital Fund. We also thank the T.J. Martell Foundation and the National Colorectal Cancer Research Alliance for additional support.

\section{References}

Breyer, R.M., Bagdassarian, C.K., Myers, S.A., and Breyer, M.D. 2001. Prostanoid receptors: Subtypes and signaling. Ann. Rev. Pharmacol. Toxicol. 41: 661-690.

Buchanan, F.G., Wang, D., Bargiacchi, F., and DuBois, R.N. 2003. Prostaglandin E2 regulates cell migration via the intracellular activation of the epidermal growth factor receptor. J. Biol. Chem. 278: 35451-35457.

Carreira-Barbosa, F., Concha, M.L., Takeuchi, M., Ueno, N., Wilson, S.W., and Tada, M. 2003. Prickle 1 regulates cell movements during gastrulation and neuronal migration in zebrafish. Development 130: 4037-4046.

Cha, Y.I., Kim, S.H., Solnica-Krezel, L., and DuBois, R.N. 2005a. Cyclooxygenase-1 signaling is required for vascular tube formation during development. Dev. Biol. 282: 274-283.

Cha, Y.I., Solnica-Krezel, L., and DuBois, R.N. 2005b. Fishing for prostanoids: Deciphering the developmental functions of cyclooxygenase-derived prostaglandins. Dev. Biol. (in press).

Chan, J., Bayliss, P.E., Wood, J.M., and Roberts, T.M. 2002. Dissection of angiogenic signaling in zebrafish using a chemical genetic approach. Cancer Cell 1: 257-267.

Davis, D.L., Pakrasi, P.L., and Dey, S.K. 1983. Prostaglandins in swine blastocysts. Biol. Reprod. 28: 1114-1118.

Dey, S.K., Chien, S.M., Cox, C.L., and Crist, R.D. 1980. Prostaglandin synthesis in the rabbit blastocyst. Prostaglandins 19: 449-453.

Dinchuk, J.E., Car, B.D., Focht, R.J., Johnston, J.J., Jaffee, B.D., Covington, M.B., Contel, N.R., Eng, V.M., Collins, R.J., Czerniak, P.M., et al. 1995. Renal abnormalities and an altered inflammatory response in mice lacking cyclooxygenase II. Nature 378: 406-409.

Dumstrei, K., Mennecke, R., and Raz, E. 2004. Signaling pathways controlling primordial germ cell migration in zebrafish. J. Cell Sci. 117 (Pt 20): 4787-4795.

Fujino, H., Xu, W., and Regan, J.W. 2003. Prostaglandin E2 induced functional expression of early growth response factor-1 by EP4, but not EP2, prostanoid receptors via the phosphatidylinositol 3-kinase and extracellular signal-regulated kinases. J. Biol. Chem. 278: 12151-12156.

Grosser, T., Yusuff, S., Cheskis, E., Pack, M.A., and FitzGerald, 
G.A. 2002. Developmental expression of functional cyclooxygenases in zebrafish [see comment]. Proc. Natl. Acad. Sci. 99: 8418-8423.

Gupta, R.A. and DuBois, R.N. 2001. Colorectal cancer prevention and treatment by inhibition of cyclooxygenase-2. Nat. Rev. Cancer 1: 11-21.

Jessen, J.R., Topczewski, J., Bingham, S., Sepich, D.S., Marlow, F., Chandrasekhar, A., and Solnica-Krezel, L. 2002. Zebrafish trilobite identifies new roles for Strabismus in gastrulation and neuronal movements. Nat. Cell Biol. 4: 610-615.

Katso, R., Okkenhaug, K., Ahmadi, K., White, S., Timms, J., and Waterfield, M.D. 2001. Cellular function of phosphoinositide 3-kinases: Implications for development, homeostasis, and cancer. Ann. Rev. Cell Dev. Biol. 17: 615-675.

Kimmel, C.B., Ballard, W.W., Kimmel, S.R., Ullmann, B., and Schilling, T.F. 1995. Stages of embryonic development of the zebrafish. Dev. Dyn. 203: 253-310.

Kobayashi, T. and Narumiya, S. 2002. Function of prostanoid receptors: Studies on knockout mice. Prostaglandins Other Lipid Mediat. 68-69: 557-573.

Kobayashi, M., Toyama, R., Takeda, H., Dawid, I.B., and Kawakami, K. 1998. Overexpression of the forebrain-specific homeobox gene six3 induces rostral forebrain enlargement in zebrafish. Development 125: 2973-2982.

Krauss, S., Johansen, T., Korzh, V., and Fjose, A. 1991a. Expression of the zebrafish paired box gene pax[zf-b] during early neurogenesis. Development 113: 1193-1206.

Krauss, S., Johansen, T., Korzh, V., Moens, U., Ericson, J.U., and Fjose, A. 1991b. Zebrafish pax[zf-a]: A paired box-containing gene expressed in the neural tube. EMBO J. 10: 3609-3619.

Langenbach, R., Morham, S.G., Tiano, H.F., Loftin, C.D., Ghanayem, B.I., Chulada, P.C., Mahler, J.F., Lee, C.A., Goulding, E.H., Kluckman, K.D., et al. 1995. Prostaglandin synthase 1 gene disruption in mice reduces arachidonic acidinduced inflammation and indomethacin-induced gastric ulceration. Cell 83: 483-492.

Langenbach, R., Loftin, C.D., Lee, C., and Tiano, H. 1999. Cyclooxygenase-deficient mice. A summary of their characteristics and susceptibilities to inflammation and carcinogenesis. Ann. NY Acad. Sci. 889: 52-61.

Leopoldt, D., Hanck, T., Exner, T., Maier, U., Wetzker, R., and Nurnberg, B. 1998. G $\beta \gamma$ stimulates phosphoinositide 3-kinase- $\gamma$ by direct interaction with two domains of the catalytic p110 subunit. J. Biol. Chem. 273: 7024-7029.

Lin, F., Sepich, D.S., Chen, S., Topczewski, J., Yin, C., SolnicaKrezel, L., and Hamm, H. 2005. Essential roles of G $\alpha 12 / 13$ signaling in distinct cell behaviors driving zebrafish convergence and extension gastrulation movements. J. Cell Biol. 169: 777-787.

Marlow, F., Topczewski, J., Sepich, D., and Solnica-Krezel, L. 2002. Zebrafish Rho kinase 2 acts downstream of Wnt11 to mediate cell polarity and effective convergence and extension movements. Curr. Biol. 12: 876-884.

Montero, J.A., Kilian, B., Chan, J., Bayliss, P.E., and Heisenberg, C.P. 2003. Phosphoinositide 3-kinase is required for process outgrowth and cell polarization of gastrulating mesendodermal cells. Curr. Biol. 13: 1279-1289.

Myers, D.C., Sepich, D.S., and Solnica-Krezel, L. 2002a. Bmp activity gradient regulates convergent extension during zebrafish gastrulation. Dev. Biol. 243: 81-98.

$2002 \mathrm{~b}$. Convergence and extension in vertebrate gastrulae: Cell movements according to or in search of identity? Trends Genet. 18: 447-455.

Narumiya, S., Sugimoto, Y., and Ushikubi, F. 1999. Prostanoid receptors: Structures, properties, and functions. Physiol. Rev. 79: 1193-1226.
Negishi, M., Sugimoto, Y., and Ichikawa, A. 1995. Molecular mechanisms of diverse actions of prostanoid receptors. Biochim. Biophys. Acta 1259: 109-119.

Pini, B., Grosser, T., Lawson, J.A., Price, T.S., Pack, M.A., and FitzGerald, G.A. 2005. Prostaglandin E synthases in zebrafish. Arterioscler. Thromb. Vasc. Biol. 25: 315-320.

Rauch, G.J., Hammerschmidt, M., Blader, P., Schauerte, H.E., Strahle, U., Ingham, P.W., McMahon, A.P., and Haffter, P. 1997. Wnt5 is required for tail formation in the zebrafish embryo. Cold Spring Harb. Symp. Quant. Biol. 62: 227-234.

Reese, J., Paria, B.C., Brown, N., Zhao, X., Morrow, J.D., and Dey, S.K. 2000. Coordinated regulation of fetal and maternal prostaglandins directs successful birth and postnatal adaptation in the mouse. Proc. Natl. Acad. Sci. 97: 9759-9764.

Reese, J., Zhao, X., Ma, W.G., Brown, N., Maziasz, T.J., and Dey, S.K. 2001. Comparative analysis of pharmacologic and/or genetic disruption of cyclooxygenase-1 and cyclooxygenase-2 function in female reproduction in mice. Endocrinology 142: 3198-3206.

Schulte-Merker, S., Ho, R.K., Herrmann, B.G., and NussleinVolhard, C. 1992. The protein product of the zebrafish homologue of the mouse $\mathrm{T}$ gene is expressed in nuclei of the germ ring and the notochord of the early embryo. Development 116: 1021-1032.

Schulte-Merker, S., van Eeden, F.J., Halpern, M.E., Kimmel, C.B., and Nusslein-Volhard, C. 1994. no tail (ntl) is the zebrafish homologue of the mouse $\mathrm{T}$ (Brachyury) gene. Development 120: 1009-1015.

Schwindinger, W.F. and Robishaw, J.D. 2001. Heterotrimeric G-protein $\beta \gamma$-dimers in growth and differentiation. Oncogene 20: 1653-1660.

Seo, H.C., Nilsen, F., and Fjose, A. 1999. Three structurally and functionally conserved Hlx genes in zebrafish. Biochim. Biophys. Acta 1489: 323-335.

Sheng, H., Shao, J., Washington, M.K., and DuBois, R.N. 2001. Prostaglandin E2 increases growth and motility of colorectal carcinoma cells. J. Biol. Chem. 276: 18075-18081.

Smith, W.L., Garavito, R.M., and DeWitt, D.L. 1996. Prostaglandin endoperoxide $\mathrm{H}$ synthases (cyclooxygenases)-1 and -2. J. Biol. Chem. 271: 33157-33160.

Smith, W.L., DeWitt, D.L., and Garavito, R.M. 2000. Cyclooxygenases: Structural, cellular, and molecular biology. Ann. Rev. Biochem. 69: 145-182.

Solnica-Krezel, L., Stemple, D.L., Mountcastle-Shah, E., Rangini, Z., Neuhauss, S.C., Malicki, J., Schier, A.F., Stainier, D.Y., Zwartkruis, F., Abdelilah, S., et al. 1996. Mutations affecting cell fates and cellular rearrangements during gastrulation in zebrafish. Development 123: 67-80.

Stephens, L., Ellson, C., and Hawkins, P. 2002. Roles of PI3Ks in leukocyte chemotaxis and phagocytosis. Curr. Opin. Cell Biol. 14: 203-213.

Stern, C.D. 2004. Gastrulation. From cells to embryo. Cold Spring Harbor Laboratory Press, Cold Spring Harbor, NY.

Takeda, H., Miyoshi, H., Tamai, Y., Oshima, M., and Taketo, M.M. 2004. Simultaneous expression of COX-2 and mPGES-1 in mouse gastrointestinal hamartomas. Brit. I. Cancer 90: 701-704.

Topczewski, J., Sepich, D.S., Myers, D.C., Walker, C., Amores, A., Lele, Z., Hammerschmidt, M., Postlethwait, J., and Solnica-Krezel, L. 2001. The zebrafish glypican knypek controls cell polarity during gastrulation movements of convergent extension. Dev. Cell 1: 251-264.

Trebino, C.E., Stock, J.L., Gibbons, C.P., Naiman, B.M., Wachtmann, T.S., Umland, J.P., Pandher, K., Lapointe, J.M., Saha, S., Roach, M.L., et al. 2003. Impaired inflammatory and pain responses in mice lacking an inducible prostaglandin E syn- 
Cha et al.

thase. Proc. Nat1. Acad. Sci. 100: 9044-9049.

Vlahos, C.J., Matter, W.F., Hui, K.Y., and Brown, R.F. 1994. A specific inhibitor of phosphatidylinositol 3-kinase, 2-(4-morpholinyl)-8-phenyl-4H-1-benzopyran-4-one (LY294002). $I$. Biol. Chem. 269: 5241-5248.

Wang, F., Herzmark, P., Weiner, O.D., Srinivasan, S., Servant, G., and Bourne, H.R. 2002. Lipid products of PI(3)Ks maintain persistent cell polarity and directed motility in neutrophils. Nat. Cell Biol. 4: 513-518.

Wang, D., Wang, H., Shi, Q., Katkuri, S., Walhi, W., Desvergne, B., Das, S.K., Dey, S.K., and DuBois, R.N. 2004. Prostaglandin $\mathrm{E}(2)$ promotes colorectal adenoma growth via transactivation of the nuclear peroxisome proliferator-activated receptor $\delta$. Cancer Cell 6: 285-295. 


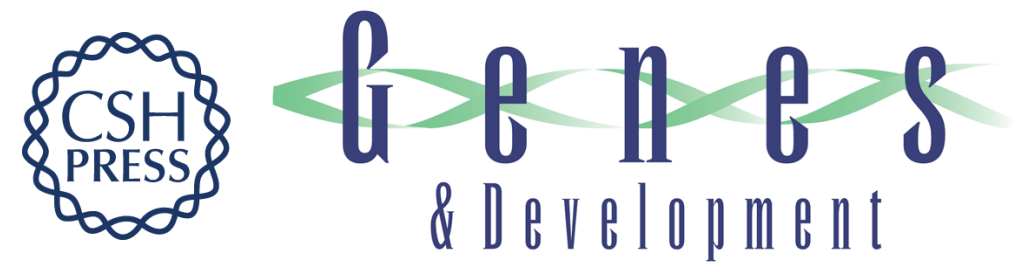

\section{Cyclooxygenase-1-derived $\mathrm{PGE}_{2}$ promotes cell motility via the G-protein-coupled EP4 receptor during vertebrate gastrulation}

Yong I. Cha, Seok-Hyung Kim, Diane Sepich, et al.

Genes Dev. 2006, 20:

Access the most recent version at doi:10.1101/gad.1374506

\section{Supplemental http://genesdev.cshlp.org/content/suppl/2005/12/30/20.1.77.DC1 Material}

Related Content

References

\section{License}

Email Alerting

Service

\section{Below the Speed Limit in the Absence of PGE2}

Sci. STKE January , 2006 2006: tw469

This article cites 48 articles, 19 of which can be accessed free at:

http://genesdev.cshlp.org/content/20/1/77.full.html\#ref-list-1

Articles cited in:

http://genesdev.cshlp.org/content/20/1/77.full.html\#related-urls

Receive free email alerts when new articles cite this article - sign up in the box at the top right corner of the article or click here.

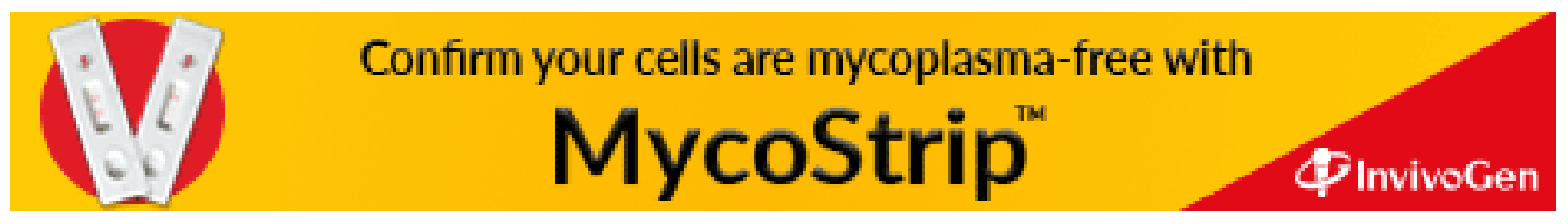

